\title{
Cartographers and geographers as toponym users, creators, and promoters. A linguistic perspective on cartographic and textual dissemination of geographical names
}

\author{
Wojciech Włoskowicz ${ }^{\text {a, * }}$ \\ ${ }^{a}$ Institute of the Polish Language, Polish Academy of Sciences, wloskowicz@gmail.com \\ * Corresponding author
}

Keywords: Toponymy, Name Dissemination, Toponym Use, Geographer, Cartographer

\begin{abstract}
:
Cartographers and geographers constitute a special and in some way privileged group of toponym users and toponym creators. The principles of establishing and using geographical names by the mentioned scientists and professionals are determined by various superior scientific, legal, political or administrative regulations that are, generally speaking, not known and not observed by the general public in their general language use. However, the use of geographical names by general public is directly or indirectly influenced by name choices and name creations made by cartographers and geographers.
\end{abstract}

Nevertheless, neither cartographers nor geographers are independent "toponymic decision-makers". Being privileged toponym users and creators, they are still only one "collective" player in a much broader and much more complex constellation of actors and factors such as: local communicative communities, already existing texts and previous maps, linguistic norm of a given language in which toponyms are meant to be used (or in which a map is to be prepared), toponymic codification of various types (official/linguistic/scientific codification), and superior (mainly legal) guidelines of language and toponymic policy of a given country. In other words: in their toponymic choices cartographers and geographers are both the influencing and the influenced ones.

The privileges which cartographers and geographers enjoy as toponym users and creators result mainly form the fact that the texts they create (and these comprise maps as well) are often automatically perceived by the general public as somehow prestigious, correct or even normative. On the other hand, the way geographers design toponyms as labels for geographical concepts differs from the "natural" and "spontaneous" way most toponyms were created or rather "came into being" in the past. The geographically and cartographically named objects are "defined" on the basis of scientific (e.g. physiographical or geological) criteria, which is obviously not the case with the geographical features "intuitively" perceived by laypeople both now and in the past.

The aim of the proposed paper is to provide an outline of a general (top)onomastic model of textual-normative dissemination of geographical names and to use this model in description and explanation of the special role cartographers and geographers play in fixing, establishing, propagating, and creating geographical names. Taking a different perspective, one could put the aim of the paper the following way as well: the goal is to describe cartographers and geographers as language users within the very specific and narrow scope of toponym use.

The onomastic model, which is to be employed, is generally based on the main theoretical concepts developed by the Prague linguistic circle and later extended and elaborated by what may be called the Polish school of normative linguistics. These concepts are: texts (language use), language norm, and language codification. Language elements (comprising proper names and thus geographical names as well) may spread or move across these three main categories which mutually influence themselves. E.g. a toponym used in spoken texts of a local community may be considered correct and counted among the normative language expressions (i.e. expressions belonging to the language norm). As such the name gets codified (fixed, established etc.) in various ways (e.g. on maps and in geographical works), which in turn results in the fact that it is used by supralocal communicative communities. 
The most generalized and simplified version of the herein proposed (top)onomastic model of textual-normative dissemination of geographical names would take the following form:

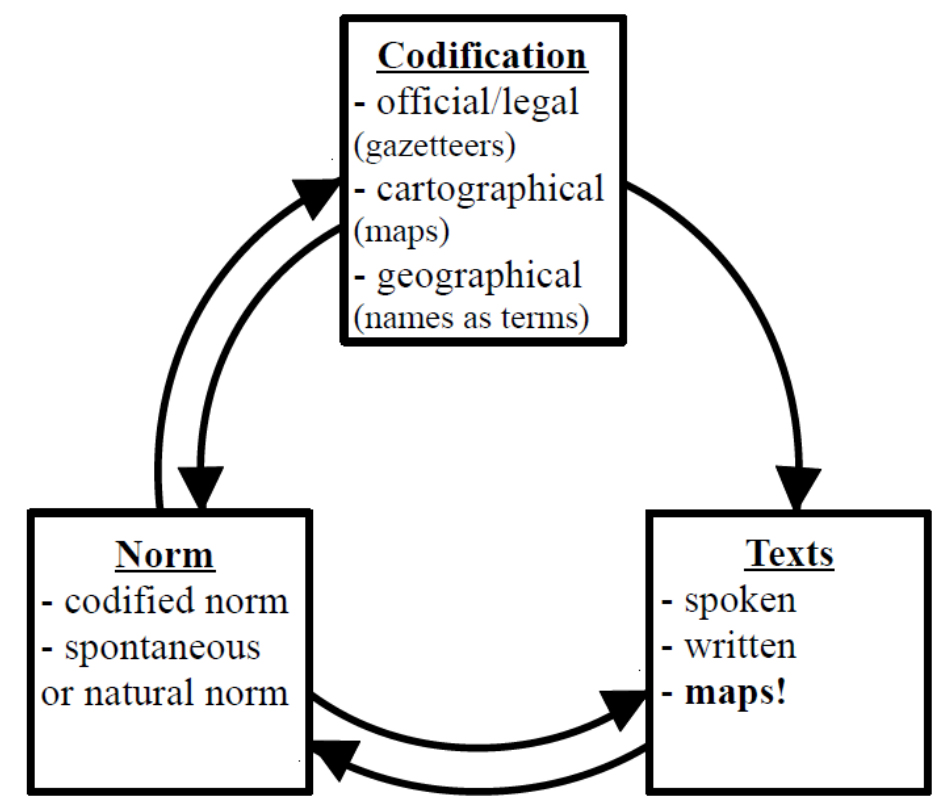

Figure 1. (Top)onomastic model of textual-normative dissemination of geographical names.

Two main conceptual oppositions need to be explained at this very point. Norm is a theoretical category, more or less an abstract set of linguistic items considered correct whereas codification is rather a "material" category that covers all items present in documents, lists etc. comprising correct items (e.g. words fixed in dictionaries). A given text may belong both to the category of codification and to the category of texts: e.g. a geography textbook or an encyclopedia are (for obvious reasons) types of written texts and a kind of codification of toponyms. The sheer presence of a toponym on a map or in a geography textbook may make it "correct" in the eye of the general public.

Within the discussed model geographical names do not live their own life as they are not any metaphysical beings. All the shifts, movements and spreads of toponyms within the proposed model and among the discussed categories are in fact done and decided by name users, in this very case by cartographers and geographers, who collect, fix, establish, create and use geographical names in their works, quite often codifying them at the same time. 